\title{
Role of MEK1 and DIAPH3 Expression in Colorectal Carcinoma
}

\author{
Abd AlRahman M. Foda ${ }^{1}$, Mohamed A. H. Ahmed ${ }^{2,3}$, Hend M. H. R. Elkalla ${ }^{4}$, Eman \\ El-Zahaf ${ }^{4}$, Heba Abdallah ${ }^{5}$, Heba M. Wagih ${ }^{2}$, Manal M. Sami ${ }^{2}$
}

${ }^{1}$ Pathology Department, Faculty of Medicine, Mansoura University, Mansoura, Egypt; ${ }^{2}$ Pathology Department, Faculty of Medicine, Suez Canal University, Ismailia, Egypt; ${ }^{3}$ Department of Pathology, East Sussex Health Care Trust, United Kingdom; ${ }^{4}$ Clinical Oncology and Nuclear Medicine Department, Faculty of Medicine, Mansoura University, Mansoura, Egypt; ${ }^{5}$ Medical Oncology, Mit Ghamr Cancer Centre, Mit Ghamr, Egypt

Background: Colorectal carcinoma (CRC) is one of the serious causes of morbidity and mortality worldwide. It is characterized by activating mutations in genes encoding Receptor Tyrosine Kinases (RAS, RAF, MEK1 or MEK2) which act as driving oncogenes. DIAPH3 deficiency has been reported to enhance cancer cell motility, invasion and metastasis and also correlates with aggressive behaviour of cancer.

Aim: To study the overexpression of MEK1 and DIAPH3 in CRC patients and their prognostic significance.

Methods: We examined the immunohistochemical expression of MEK1 and DIAPH3 using tissue microarray technique in 150 CRC specimens divided into two groups. The mucinous group (MG) included specimens of 56 mucinous adenocarcinoma and 19 signet ring cell carcinoma, while the non-mucinous group (NMG) included 75 non-mucinous adenocarcinoma specimens for comparison.

Results: MEK1 and DIAPH3 were strongly expressed in $>50 \%$ of the studied specimens. The positivity of MEK1 expression was significantly higher in NMG compared to MG $(66.7 \%$ and $34.3 \%$, respectively; $\mathrm{p}<0.001)$. In all cases, the overexpression of MEK1 was significantly associated with peri-tumoral and intra-tumoral lymphocytic response ( $\mathrm{p}=0.005$ and 0.008 , respectively). Furthermore, MEK1 overexpression showed statistically significant correlation with better OS $(\mathrm{p}=0.023)$ in the whole group of patients. The expression of DIAPH-3 did not differ significantly between NMG and MG (53.3\% and $47.1 \%$, respectively; $\mathrm{p}=0.456)$. There was strong relation between the overexpression of MEK1 and DIAPH3 $(\mathrm{p}<0.001)$.

Conclusion: The results suggest a potential synergistic role of MEK1 and DIAPH3 overexpression and the development of CRC. Further large scale studies are warranted.

Corresponding author: Hend M. Elkalla, MD; Clinical Oncology and Nuclear Medicine Department, Faculty of Medicine, Mansoura University, Mansoura, Egypt ; Email: dr.hend1113@gmail.com

Keywords: Colorectal cancer, Mucinous adenocarcinoma, MEK1, DIAPH3, TMA

Submitted: 10-Jun-2018, Revised: 16-July-2018, Accepted: 29-July-2018, Published Online: 12-August-2018

\section{INTRODUCTION}

Colorectal carcinoma (CRC) represents the $3^{\text {rd }}$ most prevalent cancer and the $4^{\text {th }}$ common cause of cancer mortality worldwide ${ }^{1}$. It accounts for $9.7 \%$ of the incidence of all cancers worldwide and $6.5 \%$ in Egypt ${ }^{2}$,

Adenomas are the precursors of most CRCs according to the well described Vogelstein adenomacarcinoma sequence, which is the most common pathogenic pathway in the tumorigenesis of colorectal cancers ${ }^{4}$. Adenomas show a progressive accumulation of genetic and epigenetic changes that are the main cause of tumor occurrence. The molecular events associated with CRC progression are due to genomic alteration causing the accumulation of somatic aberrations. It acts via a few pathways including microsatellite instability and chromosomal instability. These changes occur, either alone or in combination, leading to the development of tumors with different clinicopathological features 5 .

Adenocarcinomas represent $>90 \%$ of CRCs. Signet ring, mucinous, medullary, micropapillary, and cribriform comedo-type are pathological examples of adenocarcinomas which are well described ${ }^{6-8}$. Mucinous adenocarcinoma represnts $5 \%$ to $15 \%$ of CRC cases. It is a pathologic subtype of CRC characterized by mucin pools with floating malignant epithelium constituting $>50$ percent of the tumor and being rare leads to difficulty in studying their clinico-pathologic characteristics and clinical correlates ${ }^{9}$. The importance of this subtype lies in the differences between mucinous adenocarcinoma and non-mucinous adenocarcinoma in relation to clinico-pathologic characteristics, distinct genetic profiles, and pathogenic pathways ${ }^{10}$. Foda et al showed that mucinous adenocarcinoma subtypes of CRC carry poor prognosis and that the upregulation of HER2 and Ki67 appears to play a major role in the oncogenesis of $\mathrm{CRCs}^{8}$. 
The mitogen-activated protein kinases (MAPKs) are important components of signal transduction pathways that play crucial role in cellular processes ${ }^{11}$. Downregulation of MAPKs, specially the extracellularsignal regulated kinases 1 and 2 (ERK1/2) and the effector kinases of the Ras/Raf/MEK/ERK1/2 pathway, is activated in many cancers. Therefore, this pathway has been a potential target for the development of anticancer treatment ${ }^{12}$. MEK1 and MEK2 are dual-specificity kinases that activate ERK1 and ERK2. MEK1 and MEK2 are directly phosphorylated and activated via the RAF protein kinases ${ }^{13}$. BRAF is a serine-threonine kinase that can be activated by KRAS. It forms the highest level element of the RAF-MEK-ERK (MAPK) kinase cascade ${ }^{14}$.

MEK1 and MEK2 play important role in cancer since the recognition of their strategic role in the RASRAF-MEK-ERK pathway and the demonstration that enhancing mutations in the cDNAs encoding MEK1 and MEK2 that mimicked activation loop phosphorylation could transform cells and lead to tumorigenesis ${ }^{15}$. The first report of an amino acid-altering MEK mutation was in a lung cancer cell line in 1997. The functional consequences were not identified ${ }^{16}$. Firstly in 2007, the activating mutations in MEK1 or MEK2 were reported in cell lines of ovarian cancers ${ }^{15}$. Functioning activating mutations in MEK1 and/or MEK2 have been reported in some cancers like melanoma, CRC and lung cancer ${ }^{17}$. Genetic alterations in EGFR pathway biochemically converge to activate MEK and ERK. These pathways are adjusted by positive and negative feedback and crosstalk mechanisms ${ }^{18}$.

DIAPH3 is a diaphanous related formin 3. It is a formin homology protein with $\mathrm{FH} 1$ and $\mathrm{FH} 2$ domains signalling effectors for polarization of actin filaments and assembly. Expression of DIAPH formins in tumor cells is essential for stabilizing interphase or metaphase microtubules ${ }^{19}$. Hager et al demonstrated that DIAPH3 genomic loss is associated with many tumour types and that DIAPH3 resides at an important signalling pathway that controls the amoeboid phenotype. They showed that DIAPH3 silencing impairs endocytosis, disrupts microtubules, and stops EGFR down regulation, so enhancing EGFR activity and that of its downstream effectors ERK and MEK. DIAPH3 deficiency also enhances motility, invasion and metastasis and markedly correlates with aggressive behaviour in many cancers ${ }^{20}$.

To the best of our knowledge, the roles of MEK1 and DIAPH3 in CRCs have not been thoroughly investigated yet.

In this study, we aimed to explore the pattern of immune-histochemical overexpression of MEK1 and DIAPH3 in mucinous and non-mucinous adenocarcinoma CRC specimens. The pattern of expression was correlated with the clinicopathological features and patients' survival data.

\section{METHODS}

\section{Samples}

One hundred and fifty cases of CRC were collected from the files of surgical pathology lab at the
Gastroenterology Center, Mansoura, Egypt and who received postoperative treatment (or follow up) at Clinical Oncology and Nuclear Medicine Department, Mansoura University hospital, Mansoura, Egypt. Specimens were collected from patients who underwent surgery for pathologically confirmed CRC in the period between January 2007 and January 2012. Colorectal carcinoma specimens with mucinous histology were chosen and revised. Specimens with incomplete clinical data and those formed completely of pools of mucin with very few epithelial cells were excluded. Seventy-five cases with mucinous histology fulfilled the inclusion criteria and grouped as the mucinous group (MG). They included 56 cases of MA (mucinous component $>50 \%$ of the tumor) and 19 cases of signet ring cell carcinoma (SRCC). Another 75 cases of non-mucinous adenocarcinoma were chosen randomly for comparison from the same period and grouped as non-mucinous group (NMG). They included 47 cases of conventional adenocarcinoma (CA) and 28 cases of adenocarcinoma with mucinous component $<50 \%$ of the tumor (AMC).

Exclusion criteria included: neoadjuvant chemotherapy, incomplete clinico-pathological data, incomplete follow-up data and insufficient tissue for immunostaining in microarray sections.

The institutional research board (IRB) of Mansoura University approved the study and REMARK criteria were applied ${ }^{21}$.

\section{Clinical parameters and histopathological evaluation}

The 150 cases were reviewed as regard all the clinico-pathological data with re-examination of all their slides. This review included: age, sex, location of the tumor, size and their shape, multiplicity, histological type, tumor grading, depth of invasion (T), tumor edges (either pushing or infiltrating microscopically), lymphovascular invasion, perineural invasion, peritumoral and intra-tumoral lymphocytic infiltration, extent of neutrophilic infiltrate, nearby and distant mucosa, if the tumor is on top of adenoma or not, number of lymph node metastases $(\mathrm{N})$, distant metastasis (M), TNM staging according to the latest WHO version 6 , surgical cut margins (if infiltrated by tumor tissue or not), associated schistosomiasis and any other findings.

\section{Tissue Microarray construction}

Hematoxylin and eosin stained sections were examined to choose representative areas for TMA construction. Three manual TMA blocks were constructed using modified mechanical pencil tip method as previously described by Foda in $2013{ }^{22}$. Three representative cores of $0.8 \mathrm{~mm}$ diameter were punched out from each specimen of CRC. Cores of various normal tissues were included to serve as positive and negative controls. Sections from TMA blocks were prepared ( $4 \mu \mathrm{m}$ thickness) for routine hematoxylin and eosin and immunohistochemistry.

\section{Immunohistochemistry}

Sections from each formalin fixed paraffin embedded tissue block were cut at $5 \mu \mathrm{m}$ mounted on super frost plus slides. The slides were immunostained 
with the anti- MEK1 [p Ser221] Antibody $0.1 \mathrm{ml}$ (Novus Biologicals cat \# NBP1-41823) and the anti- DIAPH3 Antibody $0.1 \mathrm{ml}$ (Novus Biologicals cat \# NBP1-89099). The detection kit Power-Stain ${ }^{\mathrm{TM}}$ 1.0 Poly HRP DAB Kit for Mouse + Rabbit (Genemed Biotechnologies, inc, cat\#52-0017) was used. The procedure was performed according to the manufactures' instructions. The technique is fully detailed in supplementry 1.

IHC technique: Slides were incubated in an oven at $57^{\circ} \mathrm{C}$ for 10 minutes and dewaxed using warm xylene for 10 minutes and room temperature xylene for another 10 minutes. Then, they were gradually rehydrated in decreasing concentrations of alcohol followed by tap water. Sections were treated for antigen retrieval by immersing the slides in Citrate buffer $\mathrm{pH} 6$ using a conventional microwave on power $800 \mathrm{~W}$ for twenty minutes for boiling the tissue. The slides after that were washed by PBS-T (phosphate buffered saline+ 5\% Triton-X100) for two times 10 minutes each. Endogenous peroxidase activity was blocked by adding drops of alcohol based hydrogen peroxidase for 20 minutes on the slides in the humidity chamber. Another round of wash by PBS-T for 3 times $/ 3$ minutes each was performed. Following optimization of the of the primary antibodies titre, for MEK1 a concentration of 1:200 was used, and for DIAPH3 a concentration of 1:100 was used. The diluted antibodies were incubated with the sections for one hour in a humidity chamber in room temperature. Another round of wash was performed by PBS-T for 3 times/ 3 minutes each. This was followed by application of the secondary antibody and incubation for 30 minutes followed by another round of wash by PBS-T for 3 times/ 3 minutes each. DAB - chromogen was applied for 3-5 minutes after that sections were counterstained by Mayer's hematoxylin.

Evaluation of IHC: MEK1 and DIAPH3 expressions were semi-quantitatively tested for each case. Only cytoplasmic expression of both markers was considered positive and scored. Intensity of staining was graded as: 0 (negative), 1 (weak), 2 (moderate), and 3 (strong); and the percent of positive-stained cells was reported for each core. The $\mathrm{H}$-score for each core was calculated by multiplying the intensity score by the percentage of stained cells (0-300). The average H-score for each case was calculated for all the cores corresponding to each the case. The median value for MEK1 and DIAPH3 was used as a cut-off value to divide the cases into high expression group and negative/low expression group.

\section{Statistical analysis}

SPSS, version 16.0 for Windows (SPSS Inc, IBM, Chicago, Illinois) was used for data analysis. Chi-square test was used to test significant differences in categorical variables between different groups. Disease-free survival (DFS) was defined as the time from the date of surgery to the date of relapse or death, and overall survival (OS) was defined as the time from the date of surgery to the date of death. We analysed survival data using KaplanMeier method. A comparison of survival curves was performed using the log-rank test. A 2-tailed $\mathrm{p}$ value $<0.05$ was considered significant in all tests.

\section{RESULTS}

In total, 341 cases of CRC were received at surgical pathology lab of the Gastroenterology Center, Mansoura, Egypt, during the period from 2007 to 2011. According to the inclusion criteria of this study, 150 CRC specimens (44\% of those screened) were included. These included 75 cases in the MG $(22 \%$ of all screened cases) and 75 cases in the NMG (22\% of all screened cases) ${ }^{23}$. The age of patients ranged from 20 to 80 years (mean=52.7, $\mathrm{SD}=13.094$ ) and males represented $62 \%$ $(93 / 150)$ of them. The detailed clinic-pathological and histological features of the 150 CRC cases were previously described by Foda et al ${ }^{24}$. Only 145 cases were amenable for analysis due to lost tissues in the TMA cores of 5 cases from the MG.

Cytoplasmic MEK1 expression was found in 74 out of the $145 \mathrm{CRC}$ cases (51\%) using the cut-off point Hscore of 186. Two thirds of NMG cases showed MEK1 overexpression in contrast to about $34 \%$ of MG cases. NMG showed significantly higher MEK1 expression than MG (table 1).

Cytoplasmic DIAPH3 expression was detected in 73 out of the 145 CRC cases (50.3\%). 53.3\% of NMG cases and $47.1 \%$ of MG cases showed DIAPH3 overexpression which was not significantly different $(\mathrm{p}=0.456)$. Within the $\mathrm{NMG}$, there was no significant difference among CA and AMC as regard to DIAPH3 expression $(p=0.975)$. Within the $M G$, there was no significant difference among MA and SRCC in DIAPH3 expression ( $\mathrm{p}=0.790)$ (Figure 2).

Within the NMG, there was no significant difference between CA and AMC in MEK1 expression $(\mathrm{p}=0.866)$. However, MEK1was significantly overexpressed within the MG, $42.3 \%$ of mucinous adenocarcinoma cases in comparison to only $11.1 \%$ of SRCC cases ( $\mathrm{p}=0.016)$ (figure 1).

Of the 74 CRC cases that showed MEK1 overexpression, 48 cases $(64.9 \%)$ had high DIAPH3 expression. Both MEK1 and DIAPH3 overexpression were significantly correlated $(\mathrm{p}<0.001)$. Similarly, within the NMG, $62 \%$ of cases with MEK1 overexpression showed concomitant high DIAPH3 expression, which was statistically significant $(\mathrm{p}=0.033)$. Within the MG, $70.8 \%$ of cases with MEK1 overexpression showed concomitant high DIAPH3 expression, which was statistically significant $(\mathrm{p}=0.004)$ (Table 2).

In all CRC cases, MEK1 overexpression was significantly associated with peri-tumoral and intratumoral lymphocytic response $(p=0.005,0.008$ respectively). Seventy percent of cases with peritumoral lymphocytic response (Crohn's like response) and all cases with intra-tumoral lymphocytic response showed MEK1 overexpression, (Table 1). Within NMG, 75.6\% of cases with lympho-vascular emboli showed significant overexpression MEK1 $(p=0.046)$. 90\% of cases with schistosomiasis showed MEK1 overexpression, this was not significant statistically $(\mathrm{P}=0.093)$. Within $\mathrm{MG}$, age older than 40 years was associated significantly with MEK1 overexpression $(\mathrm{P}=0.021)$. 
Table 1: Correlation of MEK1 expression with histological and clinico-pathological parameters in CRC cases (the significant parameters are only shown here)

\begin{tabular}{|c|c|c|c|c|}
\hline & \multicolumn{2}{|c|}{ Cytoplasmic MEK1 expression } & \multirow[t]{2}{*}{ Chi-square $\left(\chi^{2}\right)$} & \multirow[t]{2}{*}{ P value } \\
\hline & Negative/low $(n=71)$ & High $(n=74)$ & & \\
\hline \multicolumn{5}{|c|}{ Histological type } \\
\hline NMG & $25(33.3 \%)$ & $50(66.7 \%)$ & 15.192 & $<0.001$ \\
\hline MG & $46(65.7 \%)$ & $24(34.3 \%)$ & & \\
\hline \multicolumn{5}{|c|}{ Peritumoral lymphocytic response } \\
\hline Negative & $59(56.2 \%)$ & $46(43.8 \%)$ & 7.951 & 0.005 \\
\hline Positive & $12(30.0 \%)$ & $28(70.0 \%)$ & & \\
\hline \multicolumn{5}{|c|}{ Intratumoral lymphocytic response } \\
\hline Negative & $71(51.4 \%)$ & $67(48.6 \%)$ & 7.057 & 0.008 \\
\hline Positive & 0 & $7(100.0 \%)$ & & \\
\hline
\end{tabular}

NMG: Non-mucinous group; MG: mucinous group

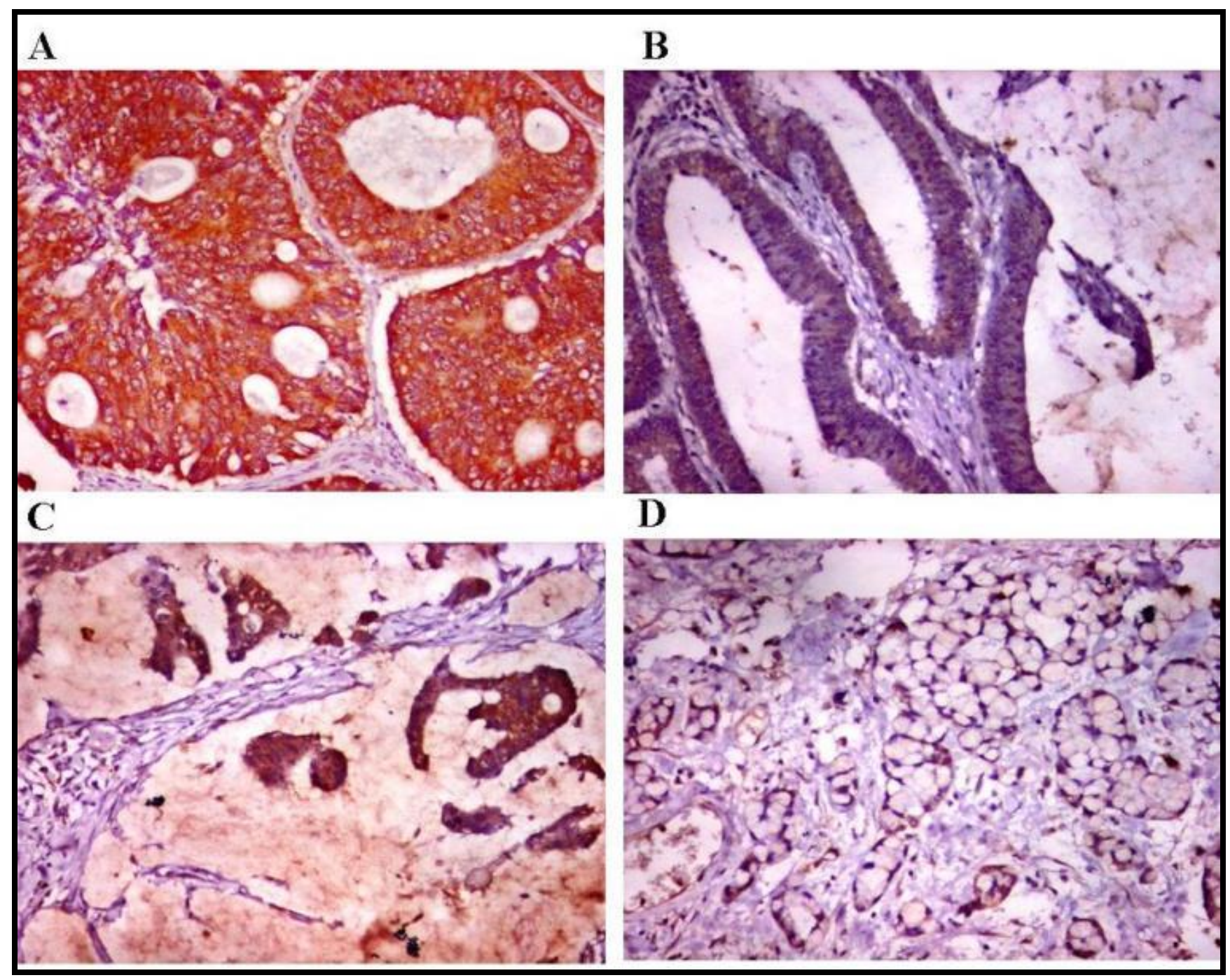

Figure 1. A: Strong diffuse MEK1 expression in a grade II ordinary colorectal carcinoma (x200); B: Focal low MEK1 expression in adenocarcinoma with mucinous component (x200); C: Strong diffuse expression in mucinous carcinoma (x200); D: Focal low MEK1 expression in signet ring cell adenocarcinoma (x200).

In all CRC cases, DIAPH3 overexpression did not show any statistically significant correlation with any of the tested variables. Within NMG, occurrence of lymphovascular emboli correlated to some extent with DIAPH3 overexpression, but this was not statistically significant $(p=0.059)$. Eighty percent of cases with schistosomiasis showed DIAPH3 overexpression, but also this was not significant statistically $(\mathrm{P}=0.069)$. Within MG, DIAPH3 overexpression was associated with neither the clinic-pathological nor the histological parameters.

At time of updated survival data for this study, all patients either relapsed or died for DFS and all patients died for OS. To investigate the possible prognostic impact of MEK1 and DIAPH3 expressions on survival of CRC cases, univariate analysis was performed for all CRC cases and for each histological type individualy (Table 3 ). 


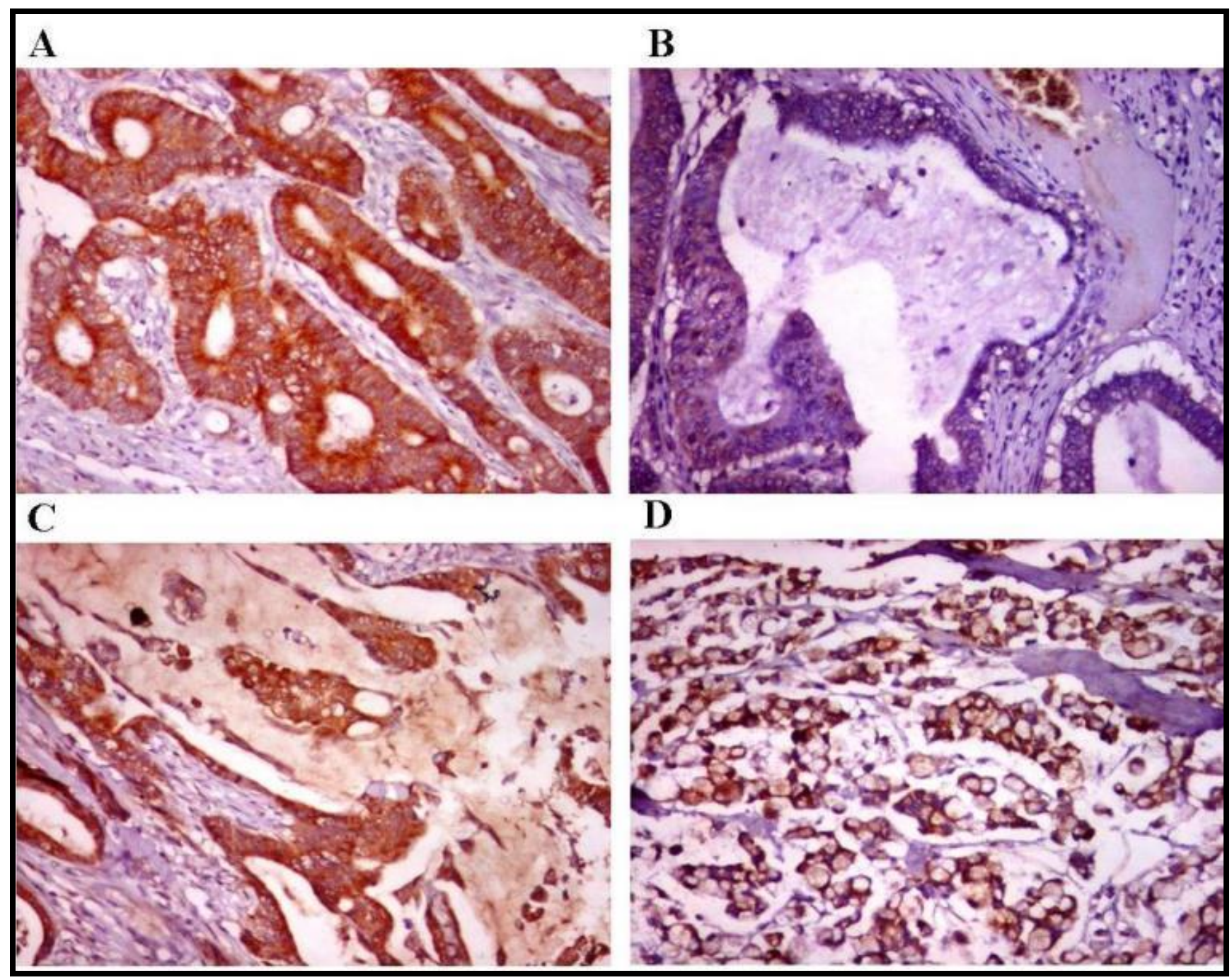

Figure 2. A: Strong diffuse DIAPH3 expression in a case of grade II ordinary CRC (x200); B: Focal low DIAPH3 expression in a case of adenocarcinoma with mucinous component (x200); C: Strong diffuse DIAPH3 expression in a case of mucinous carcinoma (x200); D: Strong diffuse DIAPH3 expression in a case of signet ring adenocarcinoma (x200).

Table 2: Correlation between MEK1 and DIAPH3 expressions in CRC cases

\begin{tabular}{|c|c|c|c|c|}
\hline & \multicolumn{2}{|c|}{ MEK1 expression } & \multirow[t]{2}{*}{ Chi-square $(\chi 2)$} & \multirow[t]{2}{*}{ P value } \\
\hline & Negative/low & High & & \\
\hline \multicolumn{5}{|c|}{ DIAPH3 expression in all CRC cases $(n=145)$} \\
\hline Negative/low & $46(64.8 \%)$ & $26(35.1 \%)$ & \multirow[t]{2}{*}{12.746} & \multirow[t]{2}{*}{$<0.001$} \\
\hline High & $25(35.2 \%)$ & $48(64.9 \%)$ & & \\
\hline \multicolumn{5}{|c|}{ DIAPH3 expression in NMG $(n=75)$} \\
\hline Negative/low & $16(64.0 \%)$ & $19(38.0 \%)$ & \multirow[t]{2}{*}{4.527} & \multirow[t]{2}{*}{0.033} \\
\hline High & $9(36.0 \%)$ & $31(62.0 \%)$ & & \\
\hline \multicolumn{5}{|c|}{ DIAPH3 expression in MG $(n=70)$} \\
\hline Negative/low & $30(65.2 \%)$ & $7(29.2 \%)$ & \multirow[t]{2}{*}{8.226} & \multirow[t]{2}{*}{0.004} \\
\hline High & $16(34.8 \%)$ & $17(70.8 \%)$ & & \\
\hline
\end{tabular}

CRC: Colorectal carcinoma ; NMG: Non-mucinous group ; MG: Mucinous group

MEK1 overexpression showed statistically significant correlation with better OS only $(\mathrm{p}=0.023)$. Furthermore, the relation of MEK1 and DIAPH3 expressions to OS and DFS of each histological suptype (CA, AMC, MA and SRCC) was also tested, and revealed no significant relations (data not shown) (Figures 3 and 4).

\section{DISCUSSION}

Despite the progress in novel treatment modalities, CRC remains one of the major health burdens all over the world. Therefore, discovering novel biological markers for targeted therapy still being of major importance in the scope of cancer treatment especially adjuvant conventional chemotherapy or targeted therapy Moreover, it is now well known that mucinous 
Table 3: Correlation of MEK1 and DIAPH3 expressions with disease-free survival and overall survival in colorectal cancer cases

\begin{tabular}{|c|c|c|c|c|c|c|}
\hline & \multicolumn{3}{|c|}{ Disease-free survival } & \multicolumn{3}{|c|}{ Overall survival } \\
\hline & $\begin{array}{l}\begin{array}{l}\text { Median } \\
\text { (months) }\end{array} \\
\end{array}$ & $95 \% \mathrm{CI}$ & P value & $\begin{array}{l}\begin{array}{l}\text { Median } \\
\text { (months) }\end{array} \\
\end{array}$ & $95 \% \mathrm{CI}$ & P value \\
\hline \multicolumn{7}{|c|}{ MEK1 expression in all CRC cases $(n=145)$} \\
\hline Negative/low expression & 25 & $19.62-30.39$ & \multirow[t]{2}{*}{0.057} & 26 & $21.05-30.96$ & \multirow[t]{2}{*}{0.023} \\
\hline High expression & 37 & $20.37-53.63$ & & 42 & $33.57-50.43$ & \\
\hline \multicolumn{7}{|c|}{ MEK1 expression in NMG $(n=75)$} \\
\hline Negative/low expression & 62 & $54-70$ & \multirow[t]{2}{*}{0.684} & 62 & $54-70$ & \multirow[t]{2}{*}{0.760} \\
\hline High expression & 53 & $33.89-72.11$ & & 53 & $35.73-70.27$ & \\
\hline \multicolumn{7}{|c|}{ MEK1 expression in MG $(n=70)$} \\
\hline Negative/low expression & 18 & $14.34-21.66$ & \multirow[t]{2}{*}{0.183} & 21 & $18.34-23.66$ & \multirow[t]{2}{*}{0.069} \\
\hline High expression & 16 & $12.48-19.52$ & & 23 & $18.2-27.8$ & \\
\hline \multicolumn{7}{|c|}{ DIAPH3 expression in all CRC cases $(n=145)$} \\
\hline Negative/low expression & 28 & $17.35-38.47$ & \multirow[t]{2}{*}{0.725} & 31 & $19.92-42.08$ & \multirow[t]{2}{*}{0.440} \\
\hline High expression & 31 & $21.24-40.76$ & & 35 & $23.85-46.16$ & \\
\hline \multicolumn{7}{|c|}{ DIAPH3 expression in NMG $(n=75)$} \\
\hline Negative/low expression & 61 & $48.69-73.31$ & \multirow[t]{2}{*}{0.676} & 61 & $48.69-73.31$ & \multirow[t]{2}{*}{0.583} \\
\hline High expression & 53 & $33.42-72.58$ & & 53 & $28.21-77.79$ & \\
\hline \multicolumn{7}{|c|}{ DIAPH3 expression in MG $(n=70)$} \\
\hline Negative/low expression & 19 & $14.74-32.26$ & \multirow[t]{2}{*}{0.582} & 21 & $17.6-24.41$ & \multirow[t]{2}{*}{0.947} \\
\hline High expression & 15 & $12.23-17.77$ & & 23 & $20.2-25.8$ & \\
\hline
\end{tabular}

CI: Confidence interval; CRC: Colorectal carcinoma ; NMG: Non-mucinous group ; MG: Mucinous group

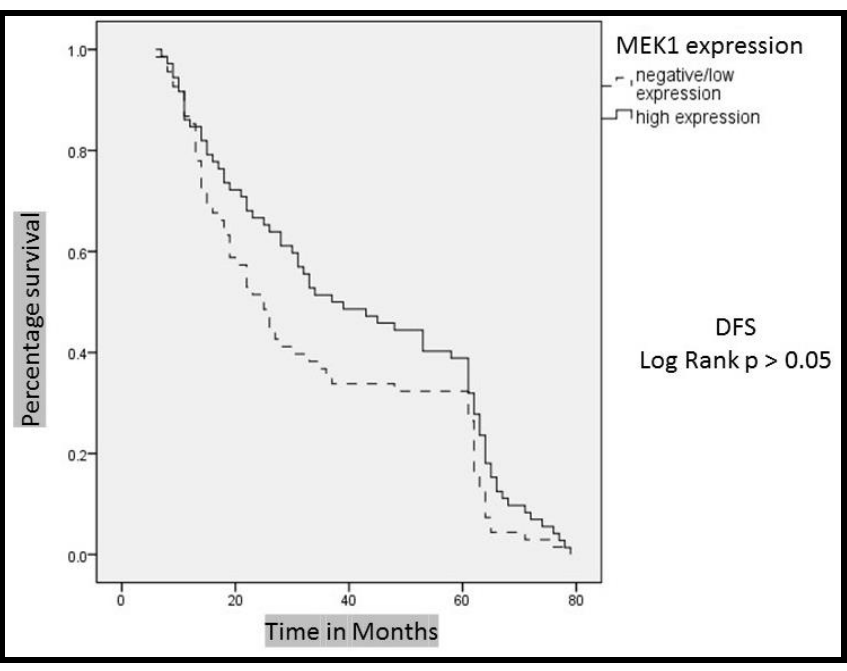

Figure 3. MEK1 expression and disease-free survival in $\mathrm{CRC}$ cases

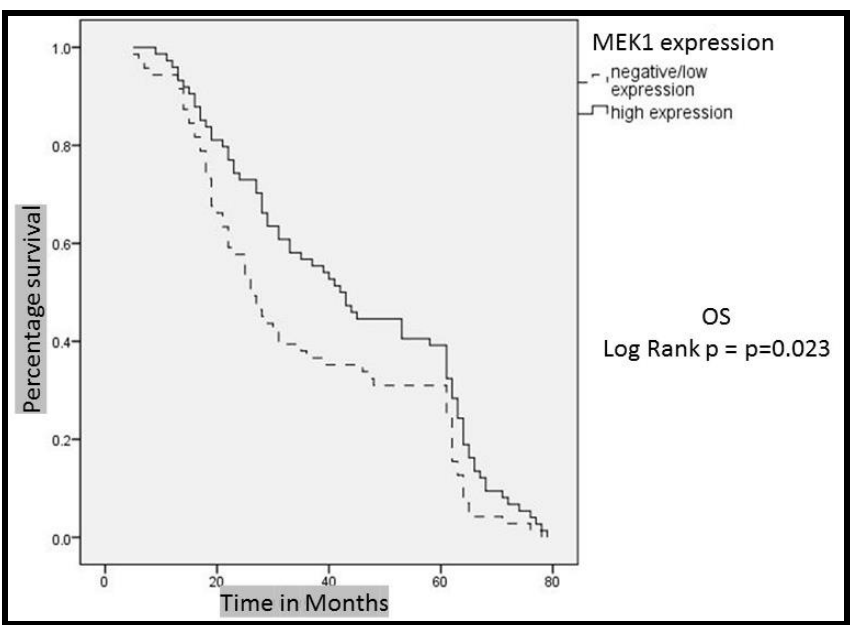

Figure 4. MEK1 expression and overall survival in CRC cases adenocarcinoma CRC is genetically dissimilar to nonmucinous adenocarcinoma variants and showed different expression of many biological markers with significant relations to survival ${ }^{25-27}$. Even CRC subgroups as AMC and SRCC showed unique genetic criteria ${ }^{28,29}$.

In the current study, we found a significant correlation between MEK-1 and DIAPH-3 expressions in both the non-mucinous and the mucinous adenocarcinomas. Additionally, the NMG showed a significantly higher expression of MEK-1 and DIAPH-3 than the MG with a significant moasic pattern in both of them. Those results are intersting, and given the very scarce data on MEK-1 and DIAPH-3 in CRC in the literature to confirm or refute the results of our study, further studies are srongly warranted.

The positivity rate of MEK1 and DIAPH-3 expression in CRC is more than $50 \%$ of the studied specimens, suggesting a potential synergistic role of MEK1 and DIAPH-3 overexpression in CRC development. Furthermore, the positivity rate of MEK1 expression is higher in the NMG but no significant difference for DIAPH-3 overexpression in the NMG and MG of the studied specimens.

The MAPK signalling pathway can be activated by extracellular signals via MEK1 which initiates the expression of proliferation and the transformation of related genes. It then will result in cellular proliferation and malignant transformation of colorectal mucosa. Increased MEK1 expression in CRC was suggested to activate the MAPK pathway that is involved in the carcinogenesis process by inducing increased cellular proliferation and anti-apoptosis ${ }^{30}$.

Bai et al demonstrated that the expression of MEK1 in grade 3 corlorectal non-mucinous adenocarcinoma and mucinous adenocarcinoma was markedly higher than in the grade 1 and grade 2 non-mucinous adenocarcinoma, which indicated that MEK1 overexpression has a potential correlation with higher tumor grade and less differentiation of tumor cells ${ }^{30}$. 
Conversely, in the current study expression of MEK1 did not exhibit any correlation with tumour grade. Additionally, we found that MEK1 is overexpressed in about $51 \%$ of the cases. Two thirds of NMG cases showed MEK1 overexpression in contrast to about $34 \%$ of MG cases. On the contrary, Bai et al that reported higher expression of MEK1 in MG than $\mathrm{NMG}^{30}$.

We demonstrated that MEK1 overexpression is significantly associated with peri-tumoral and intratumoral lymphocytic response. Seventy percent of cases with peritumoral lymphocytic response (Crohn's like response) showed MEK1 overexpression, and all cases with intra-tumoral lymphocytic response showed MEK1 overexpression, those cases are supposed to carry a poor prognosis. This is in line with the results of Bai et al who reported that MEK1 is significantly more expressed in higher tumor stages (III and IV) compared to early tumour stages and they also found that MEK1 expression was higher in metastatic lymph nodes and hepatic metastases more than in the primary tumor sites significantly ${ }^{31}$. This is suggesting that MEK1 expression could facilitate the metastasis process of CRC cells by the lymphatic or the hematogenous pathways. Additionally, adhesion and motility of CRC cells and the hematogenous and lymphatic metastasis were promoted by MEK1 activated MAPK signalling pathway ${ }^{32}$. Our results together with the results of the aforementioned studies suggest that MEK1 expression might have a potential role in CRC progression and metastasis through possible multiple mechanisms that warrant further future functional studies to assess the current findings.

We showed a strong correlation between the overexpression of MEK-1 and DIAPH-3 as more than $70 \%$ of cases with MEK-1 overexpression showed DIAPH-3 positivity and this is statistically significant in both NMG and MG. However, MEK1 and DIAPH3 expressions did not correlate significantly with OS and DFS of each histological subtype (CA, AMC, MA and SRCC). The correlation between MEK1 overexpression and better OS is difficult to interpret, however it has no correlation with DFS, and therefore this point is intriguing and needs higher scale studies to further elaborate on our findings.

There are a very few publications which addressed the role of MEK-1 and DIAPH-3 overexpression in $\mathrm{CRC}$, and to the best of our knowledge this is the first study to look at the correlation between the expression of both markers in CRC. Therefore, we recommend further studies to further scrutinize the possible joint functional attributes of MEK-1 and DIAPH-3 in CRC initiation and progression and additionally patients' prognosis.

\section{Conclusion}

The studied CRC cases showed overexpression of both MEK1 and DIAPH3 with strong correlation between them. MEK1, as a crucial member of the MAPK pathway, could play a synergistic role with DIAPH3 in CRC initiation and progression. However these results still need further research on a larger scale to explore the possible functional attributes of MEK1 and DIAPH3 in CRC and their role as potential prognostic factors.

\section{Conflict of interest}

None to declare

\section{REFERENCES}

1. Veruttipong D, Soliman AS, Gilbert SF, et al. Age distribution, polyps and rectal cancer in the Egyptian population-based cancer registry. World J Gastroenterol. 2012; 18(30): 3997-4003.

2. Ferlay J, Soerjomataram I, Dikshit R, Eser S, Mathers C, Rebelo M, et al. Cancer incidence and mortality worldwide: sources, methods and major patterns in GLOBOCAN 2012. Int J Cancer. 2015; 136(5): E359E386.

3. El-Bolkainy TN, Sakr MA, Nouh AA, El-Din NH. A comparative study of rectal and colonic carcinoma: demographic, pathologic and TNM staging analysis. J Egypt Natl Canc Inst. 2006; 18(3): 258-263.

4. Hill MJ, Morson BC, Bussey HJ. Aetiology of adenoma-carcinoma sequence in large bowel. Lancet. 1978; 1(8058): 245-247.

5. Rodriguez-Salas N, Dominguez G, Barderas R, et al. Clinical relevance of colorectal cancer molecular subtypes. Crit Rev Oncol Hematol. 2017; 109: 9-19.

6. Bosman FT, World Health Organization., International Agency for Research on Cancer. WHO classification of tumours of the digestive system. 4th ed. Lyon: International Agency for Research on Cancer; 2010.

7. Fleming M, Ravula S, Tatishchev SF, Wang HL. Colorectal carcinoma: Pathologic aspects. J Gastrointest Oncol. 2012; 3(3): 153-173.

8. Foda A, Abdelzaher E, Talaat I. Characterization of HER2 gene/protein and Ki67 protein expressions in colorectal carcinoma variants with relation to clinicopathological parameters and prognosis: An immunohistochemical and Fluorescence in Situ Hybridization study. Interdiscip Histopath J. 2015; 3(4): 120-128.

9. Foda AA, AbdelAziz A, El-Hawary AK, Hosni A, Zalata KR, Gado AI. Mucinous colorectal adenocarcinoma: Influence of EGFR and E-Cadherin expression on clinicopathologic features and prognosis. Appl Immunohistochem Mol Morphol. 2015; 23(7): 506-515.

10. Mekenkamp LJ, Heesterbeek KJ, Koopman M, Tol J, Teerenstra S, Venderbosch S, et al. Mucinous adenocarcinomas: poor prognosis in metastatic colorectal cancer. Eur J Cancer. 2012; 48(4): 501-509.

11. Chen Z, Gibson TB, Robinson F, et al. MAP kinases. Chem Rev. 2001; 101(8): 2449-2476.

12. Redwan IN, Dyrager C, Solano C, et al. Towards the development of chromone-based MEK1/2 modulators. Eur J Med Chem. 2014; 85: 127-138.

13. Yoon S, Seger R. The extracellular signal-regulated kinase: multiple substrates regulate diverse cellular functions. Growth Factors. 2006; 24(1): 21-44.

14. Morkel M, Riemer P, Blaker H, Sers C. Similar but different: distinct roles for KRAS and BRAF oncogenes in colorectal cancer development and therapy resistance. Oncotarget. 2015; 6(25): 20785-20800.

15. Caunt CJ, Sale MJ, Smith PD, Cook SJ. MEK1 and MEK2 inhibitors and cancer therapy: the long and winding road. Nat Rev Cancer. 2015; 15(10): 577-592.

16. Bansal A, Ramirez RD, Minna JD. Mutation analysis of the coding sequences of MEK-1 and MEK-2 genes in human lung cancer cell lines. Oncogene. 1997; 14(10): 1231-1234.

17. Murugan AK, Dong J, Xie J, Xing M. MEK1 mutations, but not ERK2 mutations, occur in melanomas and colon 
carcinomas, but none in thyroid carcinomas. Cell Cycle. 2009; 8(13): 2122-2124.

18. De Luca A, Maiello MR, D'Alessio A, Pergameno M, Normanno $\mathrm{N}$. The RAS/RAF/MEK/ERK and the PI3K/AKT signalling pathways: role in cancer pathogenesis and implications for therapeutic approaches. Expert Opin Ther Targets. 2012; 16 (Suppl 2): S17-S27.

19. Lin YN, Windhorst S. Diaphanous-related formin 1 as a target for tumor therapy. Biochem Soc Trans. 2016; 44(5): 1289-1293.

20. Hager MH, Morley S, Bielenberg DR, et al. DIAPH3 governs the cellular transition to the amoeboid tumour phenotype. EMBO Mol Med. 2012; 4(8): 743-760.

21. McShane LM, Hayes DF. Publication of tumor marker research results: the necessity for complete and transparent reporting. J Clin Oncol. 2012; 30(34): 42234232.

22. Foda AA. No-cost manual method for preparation of tissue microarrays having high quality comparable to semiautomated methods. Appl Immunohistochem Mol Morphol. 2013; 21(3): 271-274.

23. Foda AA, El-Hawary AK, Abdel Aziz A, Hosni A, Zalata KR, Gado AI. Rare mucinous colorectal Adenocarcinoma: analysis of the epidemiological factors in relation to survival in Egyptian patients. Am J Cancer Epidemiol Prev. 2014; 2(1): 10-19.

24. Foda AA, El-Hawary AK, Abdel-Aziz A. Differential expression of matrix metalloproteinase-13 in mucinous and nonmucinous colorectal carcinomas. Ann Diagn Pathol. 2013; 17(4): 347-351.

25. Zalata KR, Elshal MF, Foda AA, Shoma A. Genetic dissimilarity between primary colorectal carcinomas and their lymph node metastases: ploidy, p53, bcl-2, and c-myc expression-a pilot study. Tumour Biol. 2015; 36(8): 6579-6584

26. Foda AA, El-Hawary AK, Hamed H. Aberrant expression of calretinin, D2-40 and mesothelin in mucinous and nonmucinous colorectal carcinomas and relation to clinicopathological features and prognosis. Pathol Oncol Res. 2016; 22(4): 725-732.

27. Foda AA, Mohammad MA, Abdel-Aziz A, El-Hawary AK. Relation of glypican-3 and E-cadherin expressions to clinicopathological features and prognosis of mucinous and non-mucinous colorectal adenocarcinoma. Tumour Biol. 2015; 36(6): 4671-4679.

28. Foda AA, El-Hawary AK, Aziz AA. Colorectal adenocarcinoma with mucinous component: relation of MMP-13, EGFR, and E-cadherin expressions to clinicopathological features and prognosis. APMIS. 2015; 123(6): 502-508.

29. Foda AAM, Aziz AA, Mohamed MA. Colorectal signet ring cell carcinoma: Influence of EGFR, E-cadherin and MMP-13 expression on clinicopathological features and prognosis. Ann Diagn Pathol. 2018; 32: 41-46.

30. Bai X, Yu B, Su H, Du J, Yuan Q. Expression of MEK1 in the colorectal cancer and its clinical implication. ChinGer J Clin Oncol 2012; 11(3): 142-145.

31. Shama J, Garcia-Medina R, Pouyssegur J, Vial E. Major contribution of MEK1 to the activation of ERK1/ERK2 and to the growth of LS174T colon carcinoma cells. Biochem Biophys Res Commun. 2008; 372(4): 845-849.

32. Leicht DT, Balan V, Kaplun A, et al. Raf kinases: function, regulation and role in human cancer. Biochim Biophys Acta. 2007; 1773(8): 1196-1212. 